\title{
Application of medicinal plants: from past to present
}

\section{Opinion}

Medicinal plants have been a matter of special concern to people due to their therapeutic use from the past. Medicinal plants have unique and valuable properties and so have been of high interest to date. Essential oils derived from plants are used as perfumes or flavors in foods and beverages as well as herbal medicines for a long time. Historical discoveries made in India, China, Egypt and Iran show that essences are used in various ways by the people. As an example in ancient Egypt, oil was extracted by steaming. Later, the Greeks and Romans used distillation for extraction, and placed a special place for aromatic plants. With the rise of Islamic civilization, the methods of extraction of oils accelerated their evolution. In the Renaissance, Europeans, with the advancement of knowledge, improved their extraction techniques and carried out research on essential oils. Scientific research reveals the importance of the use of medicinal plants. By integrating their essential oils and justifying their therapeutic properties, scientists have been motivated to expand their cultivation and create a competitive market for them. Despite the fact that only 100 herbs are known for their essential oils, more than 2000 species of herbs are scattered in more than 60 families such as Umbelliferae, Mentha and Composite, which can be used to produce essential oils. There are 3000 types of essential oils, of which approximately 300 are of commercial importance and are offered on global markets. Essences have created many agricultural industries and also have the potential to be used in other industries such as food, pharmaceutical, perfumes and cosmetics. The production and consumption of essential oils are rising rapidly in the world, although with high prices due to the fact that a large amount of plants are needed to produce a small amount of oil, but the production of essential oils is still increasing. Therefore, their functional properties dominated their prices. The annual production of essential oils is estimated to be from 40 to 60 thousand tons, accounting for more than seven hundred million dollars. Most of the oils produced in the industry come from oranges, eucalyptus, lily of the valley, pepper and lemon, but lavender, chamomile, pepper, tea, eucalyptus, geranium, jasmine, rose, lemon, rosemary, frankincense and sandalwood are more homegrown. Countries that have the largest share of world-class essential oils include Brazil, China, America, Indonesia, India and Mexico. Large consumers include Britain, Japan, the United States and the European Union, especially Germany and France.

Due to the increase in drug resistance in microorganisms, the appropriate solution in this matter is to replace the material with a
Volume I Issue 3 - 2017

\author{
Razzagh Mahmoudi \\ Department of Food Hygiene and Safety, University of Medical \\ Sciences, Iran \\ Correspondence: Razzagh Mahmoudi, Department of Food \\ Hygiene and Safety, University of Medical Sciences, Qazvin, Iran, \\ Tel +98 (0)283336 958I, Fax +9802833345862, \\ Email r.mahmodi@yahoo.com
}

Received: June 23, 2017 | Published: June 26, 2017

suitable and different function against microbes. Among these herbal essences are the drug substances that can be used by humans and has the least side effect. Essences have the ability to fight against a wide range of germs, monocytes and insects, due to their unique compounds. Compounds from medicinal plants in the food and cosmetics industry have a central role and have a wide range of applications. Hence, the economic importance of essential oils is inextricable and controversial. With regard to scientific research, it can be concluded that essential oils have many uses in the pharmaceutical and food industries. They also have biological, pharmacological and therapeutic potential. For example, essential oils interfere with microbial activity and in many cases cause the destruction of germs without causing adverse effects on the health of the consumer. Essences also have a wide range of antiviral activity and are also used as natural products. It seems that due to the diverse application of essential oils, especially as drug compounds, they can be used to reduce the use of synthetic antibiotics. Due to the carcinogen of many compounds that are used as preservatives in a variety of health products, cosmetics, and food to increase storage time and thus threaten the safety of consumers, the importance of using herbal essences and the value of research related to this issue is several times It does. On the other hand, the use of an essential oil has long been widely accepted, and since the essential oils have very limited side effects, they can double the potential of their industrial application.

\section{Acknowledgements}

None.

\section{Conflict of interest}

The author declares no conflict of interest. 